Originally published as:

Sens-Schönfelder, C., Bozdağ, E., Snieder, R. (2021): Local Coupling and Conversion of Surface Waves due to Earth's Rotation. Part 2: Numerical Examples. - Geophysical Journal International, 225, 1, 176-185. 


\title{
Local coupling and conversion of surface waves due to Earth's rotation. Part 2: numerical examples
}

\author{
Christoph Sens-Schönfelder ${ }^{\oplus}, 1,2$ Ebru Bozdağ ${ }^{\circledR 3}$ and Roel Snieder ${ }^{2}$ \\ ${ }^{1}$ GFZ German Research Centre for Geosciences, 14473 Potsdam, Germany. E-mail: sens-schoenfelder@gfz-potsdam.de \\ ${ }^{2}$ Center for Wave Phenomena, Colorado School of Mines, Golden, CO 80401, USA \\ ${ }^{3}$ Department of Geophysics, Colorado School of Mines, Golden, CO 80401, USA
}

Accepted 2020 December 10. Received 2020 November 25; in original form 2020 September 28

\begin{abstract}
SUMMAR Y
Rotation of the Earth affects the propagation of seismic waves. The global coupling of spheroidal and toroidal modes by the Coriolis force over time is described by normal-mode theory. The local action of the Coriolis force on the propagation of surface waves can be described by coefficients for the coupling between propagating Rayleigh and Love waves as derived by Snieder \& Sens-Schönfelder. Usin6g global wavefield simulations we show how the Coriolis force leads to coupling and conversion between both surface wave types depending on latitude, propagation direction, frequency, and local velocity structure. Surface wave coupling is most efficient for periods where the modes have similar phase velocities, a condition that is equivalent to the selection rules of the angular degree in the normal-mode framework, a phenomenon that we refer to as resonant coupling. In the time-domain, resonant coupling gradually converts energy from one wave type-Rayleigh waves or Love wave-into the other, which then propagates independently. Due to the lateral heterogeneity, the condition of equal phase velocity renders the rotational coupling location-dependent. East-west oriented ray path segments and segments at high latitudes (across the Poles) only weakly couple the fundamental mode Rayleigh and Love waves while coupling is strongest for propagation along the meridians across the equator. At $250 \mathrm{~s}$ period, where Love and Rayleigh waves have similar phase velocities, the net energy transfer from Rayleigh to Love wave reaches about 10 per cent for one orbit.
\end{abstract}

Key words: Computational seismology; Structure of the Earth; Surface waves and free oscillations; Wave propagation; Wave scattering and diffraction.

\section{INTRODUCTION}

The theory of linear elasticity predicts the independent propagation of shear waves ( $S$ waves) and compressional waves ( $P$ waves) in homogeneous non-rotating media (Landau \& Lifshitz 1959). In practice there are a number of effects that cause coupling between the two wave types during propagation in the Earth's interior. Interfaces (Ben-Menahem \& Singh 1981) and heterogeneity (Sato et al. 2012) of the subsurface cause conversion between wave types. The rotation of Earth as propagation medium exerts an additional force on moving matter - the Coriolis force. Depending on the angle of the polarization vectors of $P$ and $S$ waves with Earth's rotation axis, the Coriolis force causes a small transverse component for $P$ waves and a small longitudinal component for $S$ waves. Moreover the Coriolis force causes a slow rotation of the shear wave polarization vector akin to the motion of a Foucault pendulum (Snieder et al. 2016b,a).

Similar to $P$ and $S$ waves in the bulk, surface waves are separated into transversely polarized Love waves and vertically or longitudinally polarized Rayleigh waves (Aki \& Richards 2002) that propagate independently in a laterally homogeneous non-rotating isotropic medium. Again the lateral heterogeneous and anisotropic structure of the Earth causes conversion between Love and Rayleigh waves (Kennett 1984; Snieder 1986) and Earth's rotation couples Love and Rayleigh waves (Backus 1962).

Following the observations of Earth's eigenfrequencies after the Chilean earthquake from 1960, the effects of Earth's rotation on eigenfrequencies were investigated to first and second order in $\Omega / \omega$ (e.g. Backus \& Gilbert 1961; MacDonald \& Ness 1961). Here, $\Omega$ is the angular velocity of Earth's rotation and $\omega$ is the angular eigenfrequency. The rotation causes a shift and splitting of eigenfrequency multiplets, and rotation also perturbs the displacement fields of spheroidal and toroidal normal modes (Dahlen 1968). The spheroidal modes ${ }_{n} S_{l}$ of order $l$ couple to toroidal modes ${ }_{n} T_{l \pm 1}$ and a toroidal mode ${ }_{n} T_{l}$ of couples to spheroidal modes ${ }_{n} S_{l \pm 1}$, where the difference of one angular degree is a consequence of the selection rules (Dahlen 1968). Spheroidal multiplets couple between pairs $\left.{ }_{n} S_{l},{ }_{n} S_{l}\right)$ whereas there is no coupling among toroidal multiplets by rotation (Dahlen \& Tromp 1998). 
When the eigenfrequencies of the coupled toroidal and spheroidal modes with a difference of one angular degree are in (or close to) resonance, strong coupling occurs (Dahlen 1969), and quasidegenerate perturbation theory of the nearly degenerate eigenmodes is needed to account for this coupling. While the effects of weak rotational coupling are comparable to the effects of ellipticity and heterogeneity, the strong rotational coupling of resonant modes can dominate the other types of perturbations (Luh 1974). Zürn et al. (2000) show that for frequencies below $1 \mathrm{mHz}$ rotation is the most effective mechanism to explain vertical motion observed for toroidal modes. The influence of attenuation can be integrated with perturbations from rotation and ellipticity in the calculation of eigenfrequencies using a Galerkin procedure (Park \& Gilbert 1986). Masters et al. (1983) show observations of frequency repulsion and attenuation averaging of rotationally coupled normal modes. Time-domain observation of free oscillation amplitudes show the alternation between radial and transverse displacement components for coupled modes (Park 1990). In general, the effects of Earth's rotation on deformation are well understood in the frame of normal mode theory (Dahlen \& Smith 1975; Dahlen \& Tromp 1998).

The advantage of normal mode theory for the investigation of free oscillations and globally propagating wavefields comes at the cost of an implicit description of propagation effects. Seismological observations are represented as time series point measurements which shapes the intuitive interpretation of seismograms as the signature of propagating waves rather than a superposition of normal modes.

In particular, the effect of Earth's rotation on seismic waves is described well by coupling between the different normal modes and perturbations of the eigenfrequencies. Even though the mode-ray duality can be used to synthesize time-domain seismograms of body and surface waves from normal-mode representation by summation over normal modes (Dahlen \& Tromp 1998), the local effect of the rotational coupling on propagating waves is implicit and lacks clarity.

A formulation for the coupling of travelling Love and Rayleigh waves was derived in a companion paper (Snieder \& SensSchönfelder 2020). Their treatment leads to the derivation of coupling coefficients that describe the local action of the Coriolis force on surface waves propagating in a Cartesian coordinate system. These coupling coefficients depend on the location, polarization, propagation direction and the local phase velocity dispersion curves of Love and Rayleigh waves. This approach accounts for the local coupling between transverse and radial components of propagating surface waves along a given path. An illustration of this coupling is shown in Fig. 1.

Another time-domain comparison of seismograms with and without rotation is shown by Komatitsch \& Tromp (2002b) who focus on the numerical implementation of the Coriolis force in spectral element calculations. They conclude that the maximum effect of Earth's rotation at $120^{\circ}$ distance amounts to about 3.5 per cent of the surface wave amplitude for a source on the Pole. The seismograms derived from coupled normal mode calculations by Park \& Gilbert (1986) show a considerable imprint of Earth rotation, especially for propagation paths in the north-south direction. Narrow-band precursory phases travelling with approximately the Love wave velocity ahead of the Rayleigh wave, were generated by the Coriolis force. Park \& Gilbert (1986) note that this converted energy results in a bias of traveltime and anisotropy observations if it is not correctly accounted for.

Questions about the effects of coupling between Love and Rayleigh waves arise also in the context of the seismic ambient noise field (Ardhuin et al. 2019). The ratio between horizontal and vertical amplitudes in the ambient field is affected by both the relative excitation strength of Love and Rayleigh waves as well as by propagation effects that might either act differently on both wave types or lead to an exchange of wave energy between them.

Since the ambient seismic field is excited mostly by oceans (Longuet-Higgins 1950; Ardhuin et al. 2015) and atmosphere (Nishida et al. 2000), which are devoid of shear traction, the excitation of Love waves requires a more complex excitation mechanism than Rayleigh waves. Despite the vanishing shear traction of oceanic and atmospheric loading, Nishida et al. (2008) observed the simultaneous excitation of Love and Rayleigh waves in the Pacific. Tanimoto et al. (2015) observed significant Love wave amplitudes in the microseismic period band at station Wettzell (Germany). In fact, the observed Love wave amplitudes exceeded Rayleigh wave amplitudes outside the secondary microseismic peak at $0.2 \mathrm{~Hz}$. At low frequencies the ambient vibrations occur as Earth's free oscillations known as the 'hum' (Tanimoto 2001; Rhie \& Romanowicz 2004, 2006; Webb 2007; Deen et al. 2017) which are continuously excited by processes in the atmosphere or the oceans which are not expected to excite toroidal modes. Nonetheless, Kurrle \& WidmerSchnidrig (2008) also observe continuously excited toroidal modes of the Earth. At low frequencies the most likely mechanism for the excitation of Love waves is the interaction of ocean infragravity waves with seafloor topography (Nishida et al. 2008; Nishida 2014). In the microseismic frequency band the mechanism is less clear (Tanimoto et al. 2015). Since there is no certainty about the source processes, understanding propagation effects, such as the mode-coupling by Earth's rotation or anisotropy (e.g. Park \& Yu 1993; Laske \& Masters 1998) is essential.

The local character of the theory of Snieder \& Sens-Schönfelder (2020) for the coupling of surface waves by rotation makes their approach suitable for the study of propagation effects between particular source and receiver regions. However, their theory is developed in a Cartesian geometry and the extension to spherical geometry is heuristic. Here we perform qualitative tests of the derivations by Snieder \& Sens-Schönfelder (2020) for the coupling between Love and Rayleigh waves and demonstrate the most important consequences of Earth rotation on surface wave propagation. Comparing global wavefield simulations with and without the Coriolis force allows us to investigate effects of propagation distance, source location and propagation direction for a given rotation vector in a spherical geometry. With the simulations we focus on the $4 \mathrm{mHz}$ frequency ( $250 \mathrm{~s}$ period) band where strong coupling between Love and Rayleigh waves occurs. This frequency corresponds to coupling between the ${ }_{0} T_{31}$ and ${ }_{0} S_{32}$ normal modes.

We summarize the theory of Snieder \& Sens-Schönfelder (2020) in Section 2. In Section 3, we describe the numerical simulations that we use to simulate the wave coupling. We present computed waveforms in Section 4 before concluding in Section 5.

\section{THEORY OF COUPLING BETWEEN PROPAGATING LOVE AND RAYLEIGH WAVES BY THE CORIOLIS FORCE}

In the companion paper (Snieder \& Sens-Schönfelder 2020) we analyze the local coupling between Love and Rayleigh waves by Earth's rotation by extending the theory of Kennett (1984) for the coupling of surface wave modes to include rotation. The theory is formulated in a Cartesian coordinate system where plane surface wave modes propagate in the $x$-direction and the $z$-axis points downward. The rotation vector $\Omega=\left(\Omega_{x}, \Omega_{y}, \Omega_{z}\right)^{T}$ points in an arbitrary direction. 

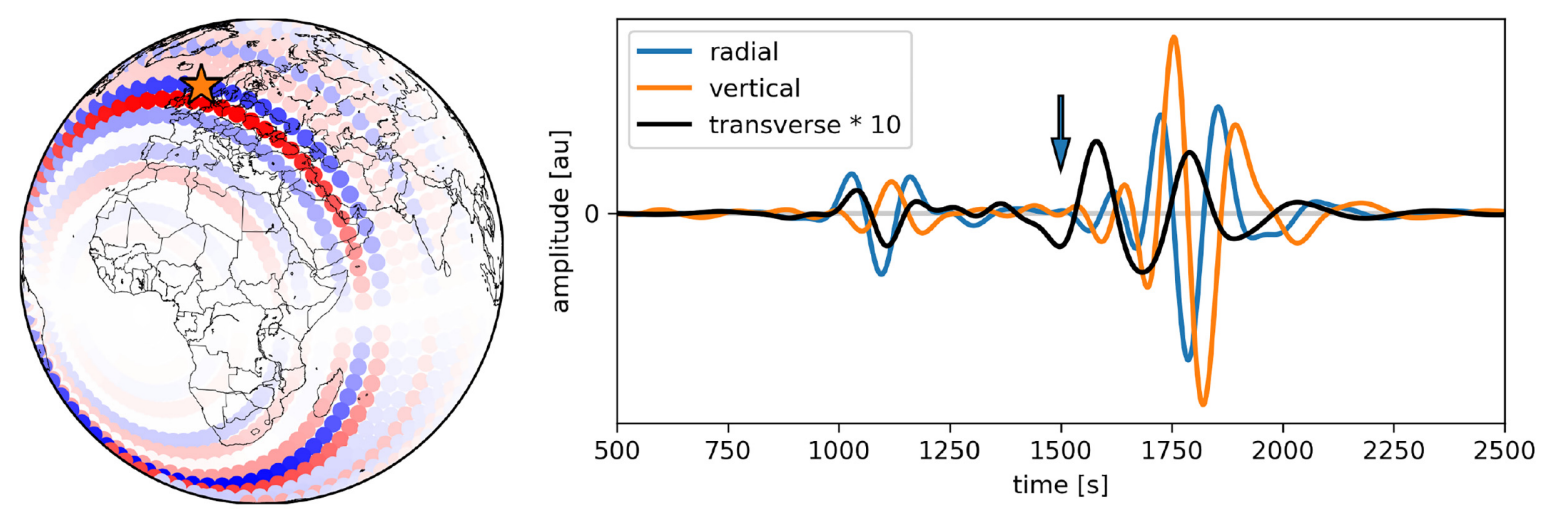

Figure 1. Excitation of Love waves by rotational coupling with Rayleigh waves. The globe shows a snapshot of the transverse motion field at $250 \mathrm{~s}$ period that is excited by coupling with Rayleigh waves due to rotation of the Earth. Without rotation no transverse motion is excited by the explosive source. The shown transverse component is magnified by a factor 10 . The transverse motion vanishes for propagation in the equatorial direction. The star at $60^{\circ} \mathrm{N}$ indicates the location for which the seismograms are shown. The arrow in the right panel indicates the time of the snapshot shown on the left. Around $1750 \mathrm{~s}$ lapse time the Rayleigh wave passes the receiver with the $\pi / 2$ phaseshift between vertical and radial components. The Love wave on the transverse component arrives earlier. Around $1000 \mathrm{~s}$ an SH wave arrives that is due to the rotation of the SV polarization induced by rotation of the Earth (Snieder et al. 2016b). Without Earth's rotation amplitudes of the transverse component, due to numerical inaccuracies, is three orders of magnitude smaller than the radial component. Synthetic seismograms were calculated using the SPECFEM3D_GLOBE package (Komatitsch \& Tromp 2002a,b).

We show in section 6 of Snieder \& Sens-Schönfelder (2020) that when the perturbation is smooth—as is the case for Earth's rotation-the coupling to backscattered surface waves is weak. When the coupling to backscattered surface waves is ignored, the displacement vector $\mathbf{w}$ of a wave propagating in $x$-direction can be represented in terms of the modes $\mathbf{w}_{r}^{0}\left(k_{r}, z\right)$ with index $r$ in a reference medium as:

$\mathbf{w}=\sum_{r} c_{r}^{+}(x) e^{i k_{r} x} \mathbf{w}_{r}^{0}\left(k_{r}, z\right)$,

where the modes $\mathbf{w}_{r}^{0}\left(k_{r}, z\right)$ and their normalization are defined in expressions (7) and (10) of Snieder \& Sens-Schönfelder (2020). The $c_{r}^{+}(x)$ are the modal coefficients for surface wave mode $r$ propagating in the positive $x$-direction. In a homogeneous reference medium these coefficients are constant. A similar expressions holds for the traction vector with the same modal coefficients (Snieder \& Sens-Schönfelder 2020). As shown by Kennett (1984), lateral heterogeneity introduces a variation of $c_{r}^{ \pm}$with position $x$. Similarly Earth's rotation perturbs the modal coefficients which are coupled by the following differential equation:

$\partial_{x} c_{q}^{+}=\sum_{r} i K_{q r} e^{i\left(k_{r}-k_{q}\right) x} c_{r}^{+}$,

where the coupling to backscattered waves has been ignored. The wavenumber of mode $q$ is denoted by $k_{q}$.

As shown in expressions (18) and (21) of Snieder \& SensSchönfelder (2020) the rotational coupling coefficients for modes propagating in the same direction are given by

$K_{q r}^{L R}=\left(K_{r q}^{R L}\right)^{*}=2 \omega \int_{0}^{\infty} \rho l_{1}^{q}\left(i \Omega_{z} r_{1}^{r}+\Omega_{x} r_{2}^{r}\right) \mathrm{d} z$,

$K_{q r}^{L L}=0 \quad, \quad K_{q r}^{R R}=-2 \omega \Omega_{y} \int_{0}^{\infty} \rho\left(r_{2}^{q} r_{1}^{r}+r_{1}^{q} r_{2}^{r}\right) \mathrm{d} z$.

In these expressions $K_{q r}^{R L}$ gives, for example, the coupling of Love mode $r$ to Rayleigh mode $q$. In these expressions $r_{1}, r_{2}$ and $l_{1}$ are the Rayleigh wave and Love wave displacement eigenfunctions as defined in Aki \& Richards (2002) that are normalized with the condition (10) of Snieder \& Sens-Schönfelder (2020). Because $K_{q r}^{L L}=0$, rotation does not introduce coupling between Love wave modes. Love and Rayleigh modes are coupled by components of the rotation vector that are vertical $\left(\Omega_{z}\right)$ and in the propagation direction $\left(\Omega_{x}\right)$. There is coupling between different Rayleigh wave modes by the component of the rotation vector $\left(\Omega_{y}\right)$ that is normal to the plane of particle motion in the unperturbed medium.

In eq. (3), $\Omega=\left(\Omega_{x}, \Omega_{y}, \Omega_{z}\right)^{T}$ is Earth's rotation vector in the local coordinate system whose $x$-axis is aligned with the direction of wave propagation. On a spherical Earth a surface wave propagating along a great circle has a varying direction of propagation on the sphere. Snieder \& Sens-Schönfelder (2020) show that for a surface wave propagating on a sphere with the azimuth $\psi$ of the propagation direction, measured clockwise from north and colatitude $\theta$ that the rotation vector of the Earth is given by

$\Omega_{x}=\Omega \cos \psi \sin \theta$,

$\Omega_{y}=-\Omega \sin \psi \sin \theta$,

$\Omega_{z}=-\Omega \cos \theta$,

In the numerical examples shown here, we analyse the coupling of Rayleigh to Love waves, as described by the coefficient $K_{q r}^{L R}$ in expression (3). For the fundamental mode Rayleigh and Love waves $(q=r=1)$ the contribution of $\Omega_{x}$ is equal to $2 \omega \Omega_{x} \int_{0}^{\infty} \rho l_{1} r_{2} \mathrm{~d} z$ while the contribution of $\Omega_{z}$ is equal to $2 i \omega \Omega_{x} z \int_{0}^{\infty} \rho l_{1} r_{1} \mathrm{~d} z$. For the fundamental modes for realistic earth models the Love wave eigenfunctions $l_{1}(z)$ and the vertical component Rayleigh wave eigenfunction $r_{2}(z)$ do not change sign with depth, while the horizontal component of the Rayleigh wave eigenfunction $r_{1}(z)$ does change sign with depth (Aki \& Richards 2002). This means that the contribution of $\Omega_{z}$ and $\Omega_{x}$ to $K_{11}^{L R}$ satisfies

$\left|\frac{\text { contribution of } \Omega_{z}}{\text { contribution of } \Omega_{x}}\right|=\left|\frac{\int_{0}^{\infty} \rho l_{1} r_{1} \mathrm{~d} z}{\int_{0}^{\infty} \rho l_{1} r_{2} \mathrm{~d} z}\right|<1$.

Ignoring the contribution of $\Omega_{z}$ implies that

$K_{11}^{L R} \approx 2 \omega \Omega_{x} \int_{0}^{\infty} \rho l_{1} r_{2} \mathrm{~d} z$.

In summary Earth's rotation has the following imprint on surface waves:

(i) a wavenumber perturbation of Rayleigh waves [section 4 of Snieder \& Sens-Schönfelder (2020)], 
(ii) coupling of surface wave modes where Rayleigh waves obtain a small transverse component while Love waves obtain a vertical/radial component [expression (44) of Snieder \& SensSchönfelder (2020)],

(iii) mode conversion: Modes with similar phase velocity convert into each other [section 5 of Snieder \& Sens-Schönfelder (2020)].

Effects (ii) and (iii) are often referred to as mode coupling, independently of whether modes have different or similar phase velocities. In the following, we demonstrate these effects on propagating surface waves using numerical wavefield simulations.

As the coefficient that describes the conversion of Rayleigh to Love waves $K_{q r}^{L R}$ is up to a difference in phase equal to the coefficient $K_{q r}^{R L}$ that describes the opposite conversion from Love to Rayleigh waves, the conversions in both directions share the same characteristics. To illustrate the conversion process we use explosive sources that only excite Rayleigh waves in radially symmetric non-rotating models, This makes it possible to observe the wave conversion that is undisturbed by the excitation of Love waves by the source. The conclusions drawn from the Rayleigh to Love wave conversion can directly be transferred to the conversion of Love to Rayleigh waves because of the symmetry of the conversion coefficients between Love and Rayleigh waves (the first identity in expression (3)).

\section{SPECTRAL-ELEMENT WAVE SIMULATIONS AND HANDLING OF CORIOLIS FORCE}

We investigate the effect of Earth's rotation on the coupling of Rayleigh and Love waves based on 3-D numerical simulations of global seismic wave propagation using the SPECFEM3D_GLOBE package (Komatitsch \& Tromp 2002a,b). This is a spectral-element solver where full anelastic wave theory can be incorporated, including topography/bathymetry, gravity (Cowling approximation), attenuation, ellipticity, rotation and the ocean load. It gives the flexibility to separate all the various effects that might couple Rayleigh and Love waves. Since our focus is on the effect of Earth's rotation we use explosive sources that only excite Rayleigh waves, perform simulations for different radially symmetric and laterally heterogeneous wave speed models and compare the wave propagation with and without Earth's rotation. The incorporation of the Coriolis force for planetary bodies in spectral-element simulations is described by Komatitsch \& Tromp (2002b). We run global simulations for periods down to approximately $10 \mathrm{~s}$ using $\mathrm{NEX}=480$, where NEX denotes the number of spectral elements on one side of each of six chunks at the surface of the cubed sphere. Despite the long periods we consider in this study, we choose a high spatial and temporal discretization to minimize numerical noise on components which should be strictly zero in theory. In the following, we present synthetic seismograms computed using radially symmetric (Sections 4.1 and 4.2) and laterally heterogeneous (Section 4.3) models.

\section{THE IMPACT OF EARTH'S ROTATION ON SYNTHETIC SEISMOGRAMS}

\subsection{Wave excitation at the equator}

In a first example we use an explosive surface source on the equator and compare the amplitudes of longitudinal and transverse wave displacements in the presence and absence of rotation for a laterally

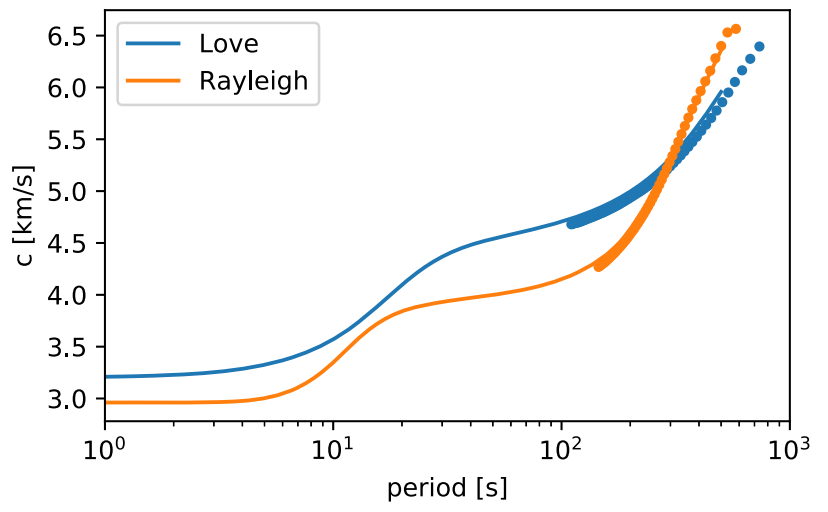

Figure 2. Phase velocity dispersion curves of fundamental mode Love and Rayleigh waves. Earth's structure is from the elastic 1-D velocity model PREM (Dziewonski \& Anderson 1981). Dots indicate great circle averaged phase velocity observations by Dziewonski \& Landisman (1970).

homogeneous Earth model. The seismogram envelope is calculated as the instantaneous amplitude of the analytical signal and its maximum in a wide time window around the surface wave arrival is used to quantify the wave amplitudes.

Fig. 1 shows the transverse amplitudes $1500 \mathrm{~s}$ after excitation by an explosion source on the equator. In numerical simulations the Preliminary Reference Earth Model (PREM, Dziewonski \& Anderson 1981) without an ocean layer is used. Since we focus on the imprint of rotation, ellipticity and self-gravitation are not taken into account. We run two sets of simulations with and without rotation and in both simulations the attenuation of PREM is used. Such an explosive source does not generate any transverse motion in the radially symmetric model used here. However, in a rotating Earth there is significant transverse energy propagating away from the equator. The traces show the arrival of $S$ waves at $1100 \mathrm{~s}$ that are caused by conversion from $P$ waves at interfaces. The transverse component, which is only generated in the simulation with rotation, is due to the Foucault pendulum-like rotation of the $S$-wave polarization (Snieder et al. 2016b).

As shown by Snieder \& Sens-Schönfelder (2020), the strongest coupling between Love and Rayleigh waves by Earth's rotation should be observed for degenerate phase speeds. In the PREM model, Love- and Rayleigh-wave phase-dispersion curves cross at a period of about $250 \mathrm{~s}$ (Fig. 2). We thus focus on the period band between 200 and $300 \mathrm{~s}$ in our seismograms.

Fig. 3 shows the amplitude of the transversely polarized wavefield as a function of epicentral distance and azimuth. The transverse wave motion vanishes for waves propagating east- and westwards along the equator whereas the transverse motion steadily increases for propagation in the north and south directions. This dependence on wave azimuth can be derived from eqs (3) and (4) which show that the real part of coupling coefficient $K^{R L}$ involves the factor $\Omega_{x}$ that is maximum for propagation along the meridians $(\psi=0$ or $\psi=180^{\circ}$ ) and zero in the zonal direction. In fact, the wave amplitude in the north-south direction is increasing with distance up to $90^{\circ}$ distance despite geometrical spreading and attenuation. The azimuthal dependence of transverse motion excitation agrees with normal mode synthetics and observations by Park (1986). Amplitudes of the transverse motion in the absence of rotation are three orders of magnitude below those shown in Fig. 3 and are attributed to numerical errors in the simulation. The radial motion in the absence of rotation does not depend on azimuth, confirming that the 


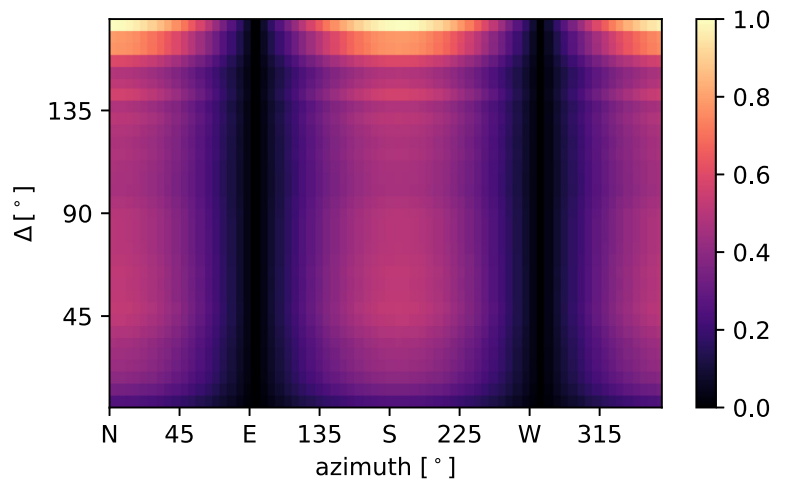

Figure 3. Normalized amplitude of the transversely polarized wave excited by an explosive source at the equator. Values are normalized to the maximum transverse amplitude. Source and antipode locations are not shown because of diverging amplitudes. The transverse motion vanishes for propagation in the east and west directions. The amplitude of the transverse component of wave propagating in the meridional direction increases with distance.

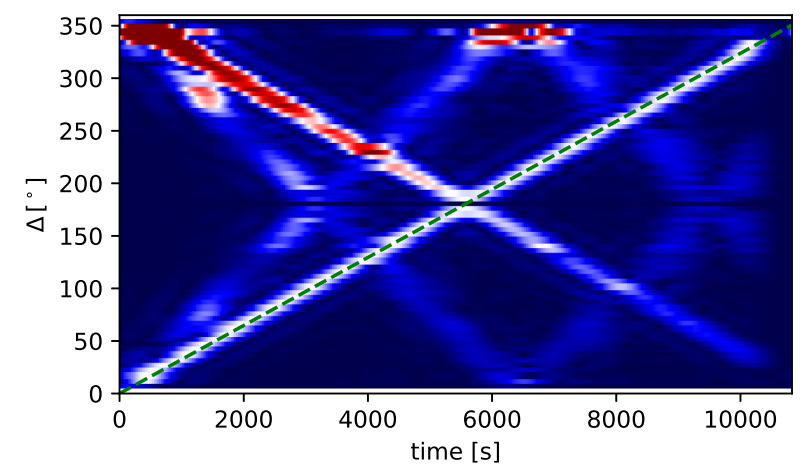

Figure 4. Radially polarized wavefield observed along the meridian of the equatorial source. Amplitudes are normalized for the amplitude of the radial component of the Rayleigh wave propagating with a group velocity of $3.6 \mathrm{~km}$ $\mathrm{s}^{-1}$. Amplitudes for $\Delta<180^{\circ}$ and $\Delta>180^{\circ}$ are identical except for the normalization that is applied for minor (R1) and major arc (R2) Rayleigh waves, respectively.

pattern of transversely polarized waves is due to the coupling by the Coriolis force.

To illustrate where the coupling occurs along the propagation path we analyse the evolution of the amplitude of the transverse motion for a full orbit along the meridian. We again use an explosive source at the equator. The amplitude of the radially polarized wavefield is shown in Fig. 4 normalized for the amplitude in a $1000 \mathrm{~s}$ long traveltime window centered around a wave propagating with the Rayleigh wave group velocity $v_{R}=3.6 \mathrm{~km} \mathrm{~s}^{-1}$ over $360^{\circ}$ distance (dashed line). The time window for the normalization therefore corresponds to the minor arc Rayleigh wave (R1) for distances $\Delta<180^{\circ}$ and to the major arc Rayleigh wave (R2) for $\Delta>180^{\circ}$. The same normalization is used for the amplitude of the transversely polarized wavefield shown in Fig. 5. White lines overlain in Fig. 5(b) indicate different velocities. Energy on the transverse component is propagating faster than $v_{R}$. A first wave train propagating at the Love wave group velocity of $v_{L}=4.2 \mathrm{~km} \mathrm{~s}^{-1}$ is generated at the source (blue patch). At distances beyond $180^{\circ}$ it is followed by a second wave train with the same group velocity $v_{L}$ that is generated at the antipode $\left(180^{\circ}\right.$ distance $)$ at the time when the Rayleigh wave passes the caustic (orange patch). This dominant excitation of transverse motion at the antipode is a consequence of energy focusing at the (a)

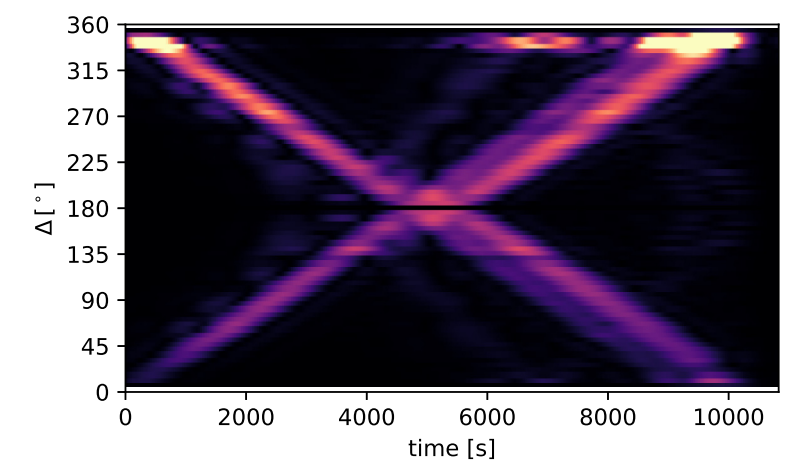

(b)

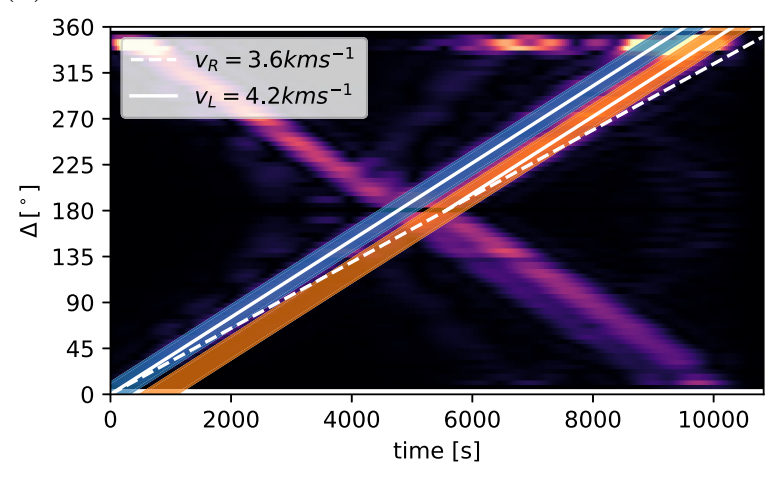

Figure 5. Amplitudes of the transversely polarized wavefield along the meridian of the equatorial source normalized for the amplitude of the Rayleigh wave propagating at $3.6 \mathrm{~km} \mathrm{~s}^{-1}$ (a). (b) Overlay of velocities and time windows used to estimate wave amplitudes. Rayleigh wave velocity is shown by the dashed line. Love wave velocity is indicated by bold lines for one wave emanating from the source and another one emanating from the antipode $\left(\Delta=180^{\circ}\right)$ at the time of the Rayleigh arrival. The blue and orange bars indicate the time windows for picking the maxima for the wave amplitude shown in Fig. 6.

caustic which leads to a concentration of transverse motion in time (Snieder \& Sens-Schönfelder 2020).

We track the envelope maximum in $600 \mathrm{~s}$ long time windows centered around the travelling Rayleigh and Love waves in Fig. 6. Transverse amplitude is measured in the two time windows indicated in Fig. 5 corresponding to waves excited at the source and the antipode location during passage of the Rayleigh wave. Fig. 6(a), displaying wave amplitudes in the period band 200-300 s, shows a rapid increase of the transverse motion amplitude reaching about 15 per cent of the amplitude of the radial motion at $45^{\circ}$ distance it decreases. This decrease is interrupted by waves focusing at the antipode. In contrast, the amplitude in the time window measuring the excitation of transverse motion by rotational coupling at the antipode increases at the antipode to about 20 per cent of the radial amplitude. In the 100-200 s period window shown in Fig. 6(b) the situation is different and the amplitude on the transverse component remains below 5 per cent due to the difference in phase velocities between Love and Rayleigh waves in this period band which prevents strong coupling and conversion (Snieder \& Sens-Schönfelder 2020).

Since the generation of Love waves by the Coriolis force occurs continuously as the Rayleigh wave propagates, the Love wave is spread out in time. This is most apparent by the two apparent Love wave trains in Fig. 5(b) that are generated at the actual source 
(a)

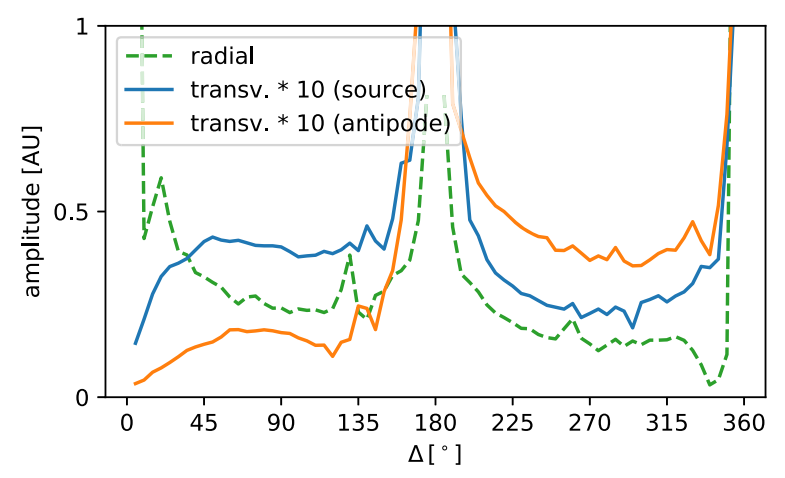

(b)

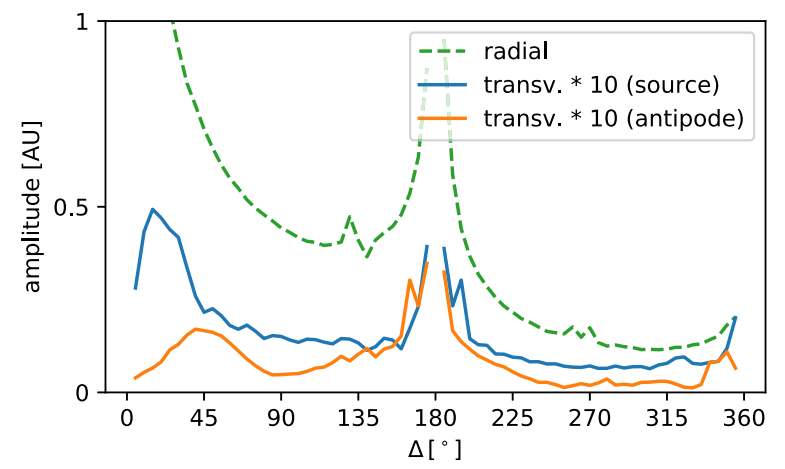

Figure 6. Comparison of wave amplitudes on radial and transverse components. (a) Period band from 200 to $300 \mathrm{~s}$ and (b) period band between 100 and $200 \mathrm{~s}$. The two curves for transverse components correspond to two waves that are excited at the source and antipode locations and propagate with Love wave velocity (see Fig 5). Note the 10-fold relative amplification of transverse amplitudes that is used for illustration purposes.

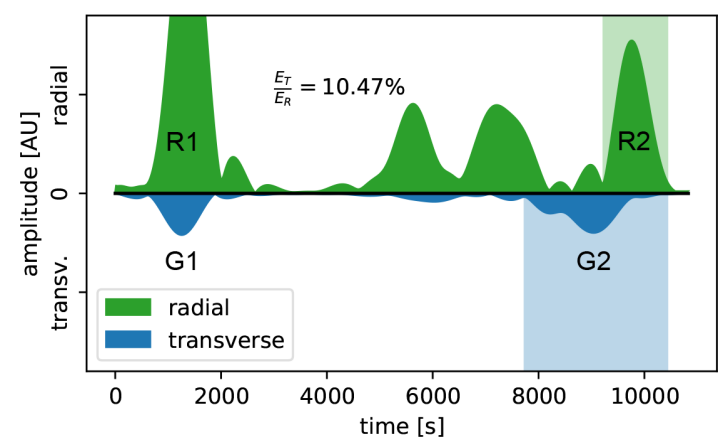

Figure 7. Envelopes of transverse and radial components at $315^{\circ}$ distance from the source. Shading in the background indicates the integration time windows for estimation of energy. G2 and R2 indicate the major arc Love and Rayleigh waves, respectively. For the radial component this corresponds to the R2 wave. The window for the transverse component starts $600 \mathrm{~s}$ before the G2 wave and ends after the R2 wave. The energy on the transverse component sums up to 10 per cent of the radial Rayleigh wave. G1 and R1 indicate the minor arc Love and Rayleigh waves with an epicentral distance of $45^{\circ}$, respectively.

and the antipode. The amplitude maximum shown in Fig. 6 alone does not suffice to compare the energy content of the transverse motion since it does not take into account the duration of Love and Rayleigh waves. Fig. 7 shows the envelopes of the transverse and radial components at a distance of $315^{\circ}$ as a function of time.
To obtain estimates of the wave energies we integrate the squared envelopes of the radial component over a time window around the $\mathrm{R} 2$ wave $[t(\mathrm{R} 2)-500 \mathrm{~s}, t(\mathrm{R} 2)+700 \mathrm{~s}]$ for the Rayleigh wave and the squared transverse envelope over a time window starting before the major arc Love wave (G2) lasting until the R2 wave has passed $[t(\mathrm{G} 2)-600, t(\mathrm{R} 2)+700]$. These time windows are indicated by the shaded time intervals in Fig. 7. Using these estimates, the energy of the transverse component is 10 per cent of the energy on the radial component in the 200-300 s period window.

\subsection{Influence of source location}

The excitation of Love waves by conversion from Rayleigh waves due to Earth's rotation depends on the amplitude of Rayleigh wave motion and the propagation direction as described by expressions (3) and (4). Coupling requires a component of Rayleigh wave propagation parallel to the rotation axis. Additionally, the focusing of the Rayleigh wave increases the Love wave excitation at the source and the antipode. For a source at the equator, the location of maximum coupling for north-south propagation coincides with the strongest focusing of the Rayleigh wave which results in dominant Love wave excitation at source and antipode locations.

The situation is different for other source locations. As shown in expression (6) the coupling from the fundamental mode Rayleigh wave to the fundamental mode Love wave depends mostly on $\Omega_{x}$. According to expression (4), at the poles, $\Omega_{x}=0$ and $\Omega_{z}= \pm \Omega$, hence according to eq. (6) the coupling of the fundamental mode Love and Rayleigh waves is weak at the poles. This means that for a source at the pole the near-source coupling between fundamental mode Rayleigh and Love waves is weak. However, as propagation distance from the polar source increases, coupling increases until the propagation direction is aligned with the rotation axis on the equator. Beyond the equator, coupling decreases again and vanishes at the caustic on the South Pole. The evolution of the maximum transverse amplitude for a polar source with distance and azimuth is shown in Fig. 8(a).

Vanishing rotational coupling at the source illustrates that the excitation of Love waves really occurs due to conversion along the way during the Rayleigh waves propagation. The time dependence of observed Love wave generation is shown in Fig. 9 which shows that the increase of Love wave amplitude is strongest at a distance of $90^{\circ}$ when Rayleigh waves cross the equator where coupling is at a maximum ( $c f$. Fig 5a).

Sources at mid latitudes have a more complex dependence of Love wave excitation on azimuth and distance (Fig. 8b). For small epicentral distances, the coupling vanishes for east-west propagation but increases with distance since rays assume an oblique angle with meridians and thus acquire a component parallel to the rotation vector.

A surface wave that leaves the source at latitude $50^{\circ} \mathrm{N}$ (Fig. 8b) with an arbitrary azimuth $\psi_{0}$ in the range $\left|\psi_{0}\right|<90^{\circ}$, travels along a great circle that is inclined with respect to the rotation axis. The propagation initially has a northward component $\left(|\psi|<90^{\circ}\right.$ in eq. (4)) before reaching the point of highest latitude and then assumes a southward component $\left(|\psi|>90^{\circ}\right)$ before crossing the source latitude $\left(50^{\circ} \mathrm{N}\right.$ in Fig. 8$)$ again. The contributions of the two segments with the northward and southward components are equal but of opposite sign. Hence the total Love wave generation is zero when the wave crosses the source latitude, which causes the transverse motion to vanish when the source and receiver are at the same latitude (the green line in Fig. 8b). 
(a)

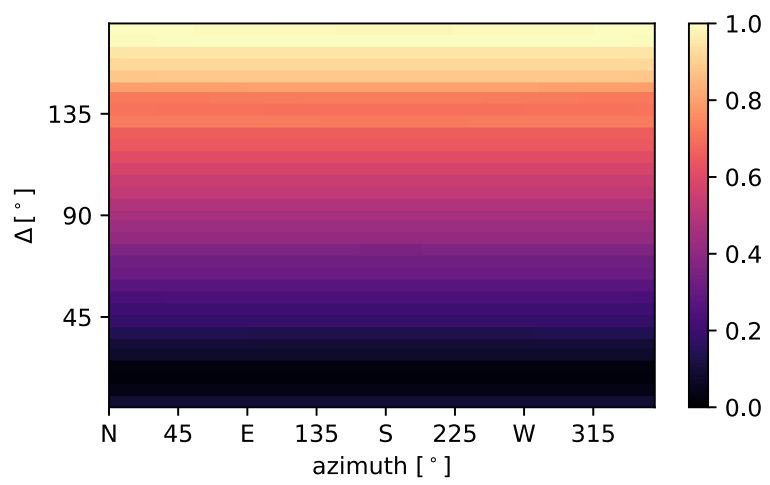

(b)

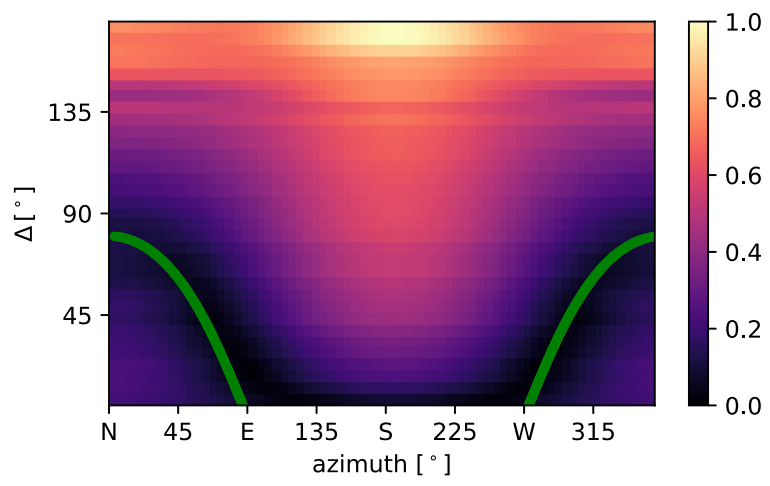

Figure 8. Normalized amplitude of the transversely polarized wave excited by a source at the pole (a) and a source at $50^{\circ} \mathrm{N}(\mathrm{b})$. The green curves in panel (b) indicate azimuth and distance along a small circle of constant latitude at $50^{\circ} \mathrm{N}$ leaving the source in the east or west direction.

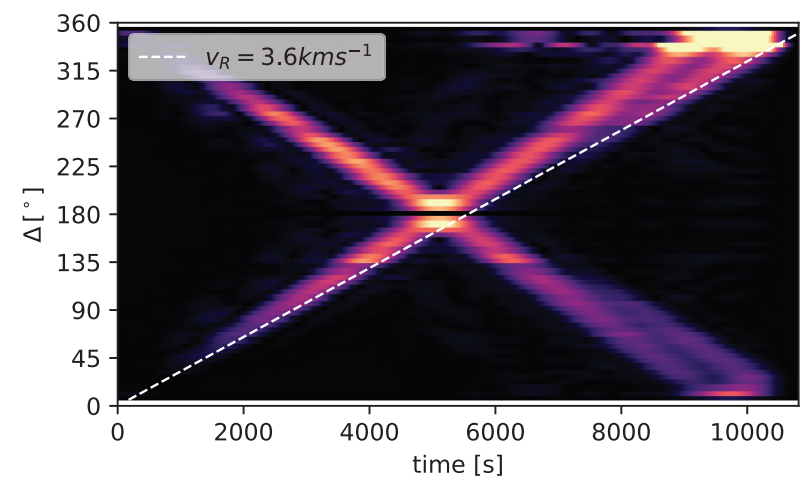

Figure 9. Envelope of the transverse component of the motion in PREM with rotation for an explosive source at the pole. This wave field has rotational symmetry leading to identical seismograms for all azimuths.

\subsection{Comparison with the effect of heterogeneity}

To assess the importance of the rotational coupling one needs to compare it to other coupling mechanisms. An obvious mechanism is the Rayleigh to Love wave coupling by lateral heterogeneity. We compare wave propagation in a rotating and non-rotating Earth in the presence of lateral heterogeneities for the 3-D global mantle model S362ANI (Kustowski et al. 2008), which is transversely isotropic in the upper mantle, together with the 3-D global crustal model Crust2.0 (Bassin et al. 2000). In this case, topography/bathymetry, (a)

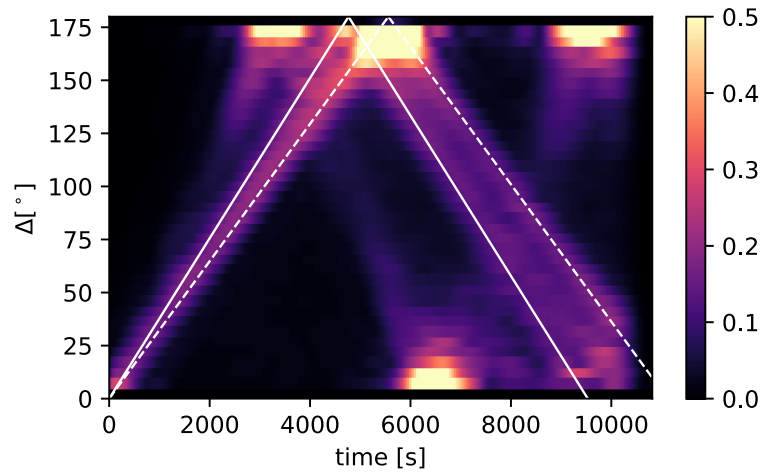

(b)

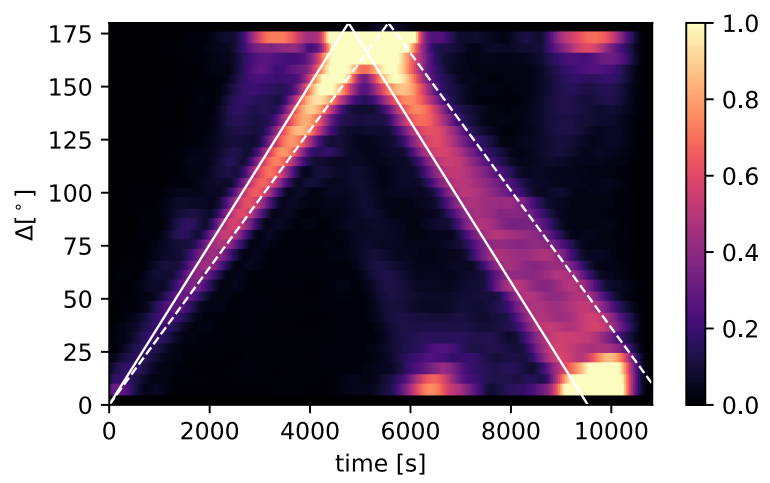

Figure 10. Envelope of the transverse components for source at North Pole in 3-D heterogeneous velocity model S362ANI. Envelopes for all azimuths are averaged. (a) without rotation and (b) with rotation. Note the different colour scale limits.

the ocean load and attenuation are considered while gravity and the ellipticity of the planet are disregarded. Beghein et al. (2008) investigated a similar situation using normal modes to study the influence of anisotropy on mode coupling. As in the simulations for the laterally homogenous earth models we kept rotation on and off to generate two sets of seismograms. We again use an explosive source located at the North Pole and investigate the $250 \mathrm{~s}$ period band of resonant coupling. To obtain representative envelope sections we average envelopes for all available azimuths. As shown in Fig. 10(a), heterogeneity causes motion on transverse components in the absence of rotation. There is transverse energy accompanying the Rayleigh wave but earlier arrivals of body waves are of similar amplitude. However in the presence of rotation the amplitudes on transverse components are more than twice as large (Fig. 10b) and dominated by the propagation of Love waves.

In Fig. 11 we quantify the effects of rotation and heterogeneity by integrating the energy on the transverse component over the surface of the Earth and comparing it to the energy on the radial components. Irrespective of the presence of rotation, the transverse energy is zero at the beginning of the simulation and increases during the wave propagation. In the presence of rotation, transverse energy grows faster and reaches close to 10 per cent of the energy of the radial component at propagation times larger than about $5000 \mathrm{~s}$. If only heterogeneity causes transverse motion the energy of the transverse motion remains at about 1 or 2 per cent of the radial energy. 


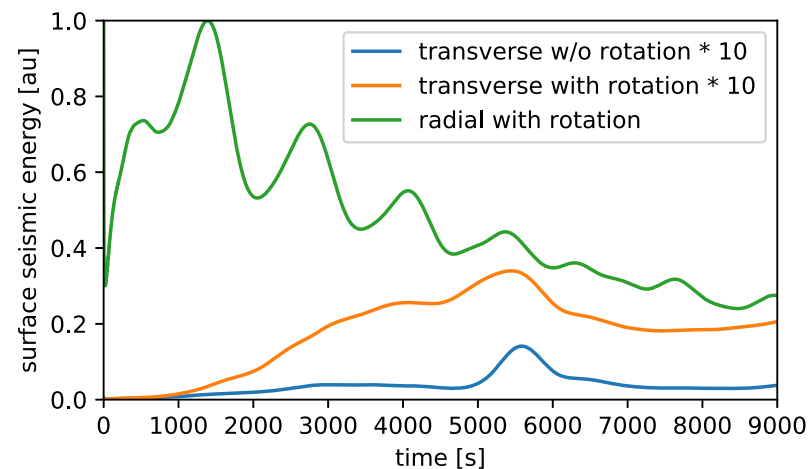

Figure 11. Seismic energy integrated over the surface of the whole Earth for radial and transverse polarization excited by an explosion at the North Pole. Wave propagation was modelled in the heterogeneous 3-D velocity model s362ani. Energy on the transverse component is significantly enhanced by Earth's rotation and reaches almost 8 per cent of radial energy beyond $180^{\circ}$ distance.

\subsection{Phase shift of Rayleigh waves}

The numerical simulations on a rotating Earth show a jump in the phase of the radial motion at the frequency where the Rayleigh waves and Love waves have the same phase velocity at a frequency of about $4 \mathrm{mHz}$. A similar shift also occurs in the normal mode frequencies of spheroidal modes (Masters et al. 1983). In this section we investigate the step in the phase of spheroidal modes as a function of propagation distance. Because we study propagating

(a)

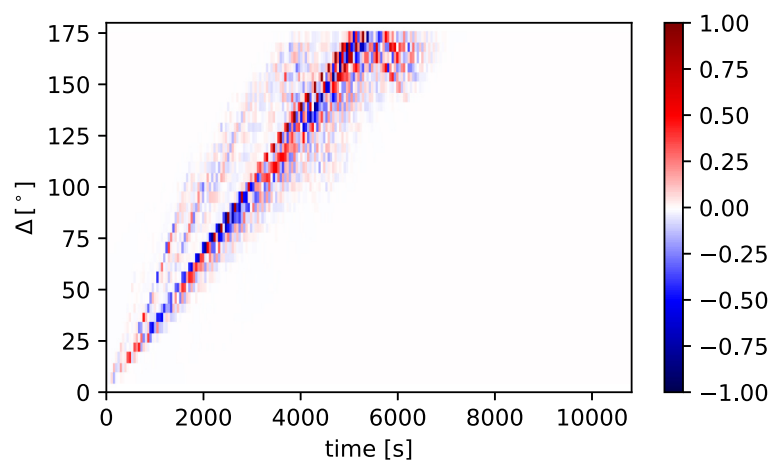

(b)

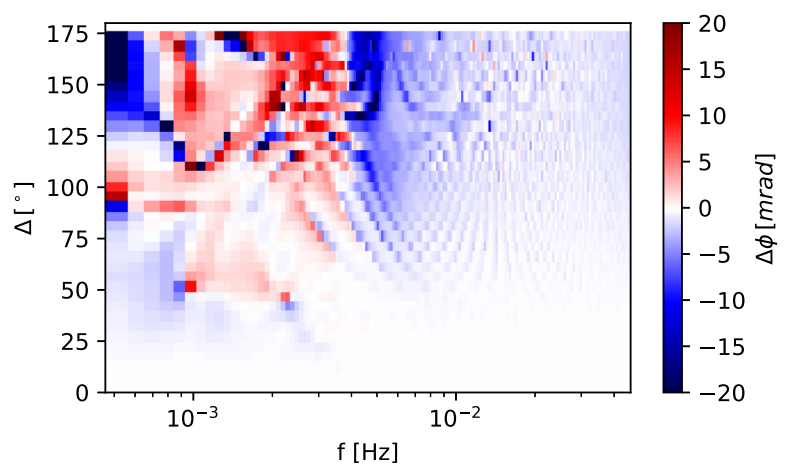

Figure 12. Phase change of the radial motion due to Earth's rotation. (a) Broad-band radial seismogram section tapered around the R1 segment of the Rayleigh wave. (b) Phase difference of the seismogram section in (a) for simulations with and without Earth's rotation. (a)

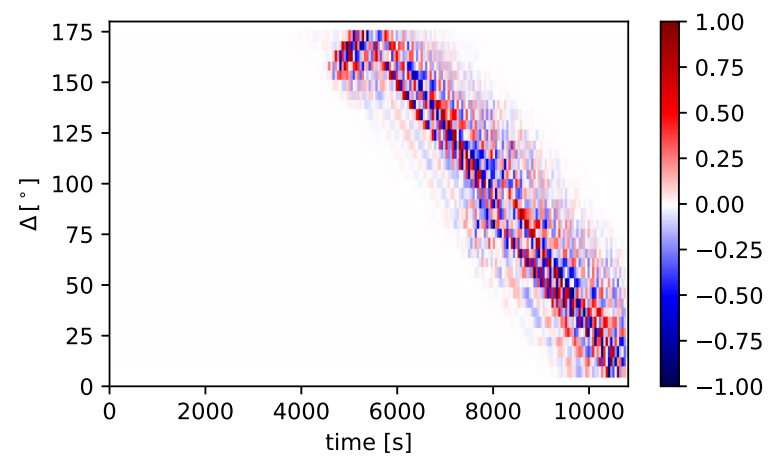

(b)

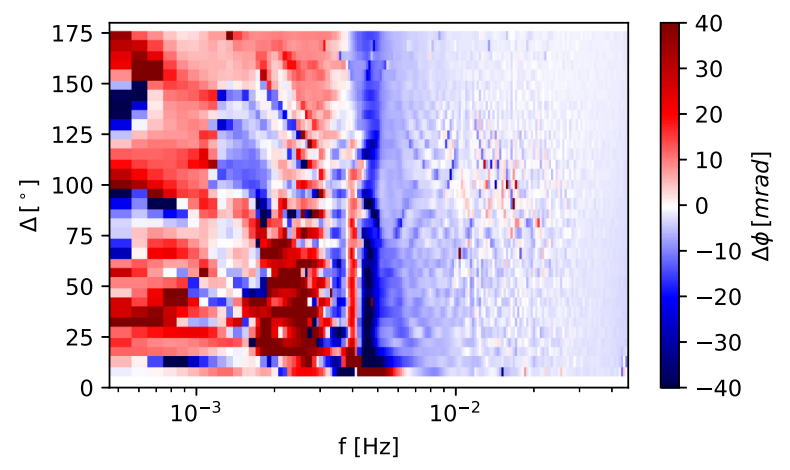

Figure 13. Same as Fig. 12 but for the R2 segment of the Rayleigh wave. Note that the propagation distance of the R2 wave increases from $\Delta=180^{\circ}$ towards $\Delta=0^{\circ}$.

surface waves, the step in the phase corresponds to a step in phase velocity.

This step in the phase can be visualized as a difference in the phase shift between numerical seismograms with and without rotation. For increasing traveltime the phase velocity step causes a growing phase difference. We illustrate this in Figs 12 and 13 in which panel (a) shows the broad-band radial seismograms tapered around R1 and R2, respectively, whereas panel (b) shows the phase difference of these seismograms for the simulations with and without rotation. A clear change in the phase difference occurs in Figs 12(b) and 13(b) at about $4 \mathrm{mHz}$ ( $250 \mathrm{~s}$ period). Fluctuations in the phase originate from interfering body waves. Note that the amplitude of the phase difference increases with traveltime. For the minor arc R1 wave (Fig. 12b) traveltime increases with distance from the source. For the major arc R2 wave (Fig. 12b) the source distance $\Delta$ decreases for increasing traveltime. Averaging over the distance ranges of the R1 and R2 waves as shown in Fig. 14 reveals a clear transition from positive phase difference below $4 \mathrm{mHz}$ period to a negative phase difference above for both R1 and R2 segments of the Rayleigh wave with a larger amplitude for R2. A phase difference of $20 \mathrm{mrad}$ corresponds to a traveltime difference of $0.8 \mathrm{~s}$ at period of $250 \mathrm{~s}$.

The jump in the phase velocities at a period of around $250 \mathrm{~s}$ can be explained as follows. As shown by Snieder \& Sens-Schönfelder (2020) the surface wave modes on a rotating Earth are close to Rayleigh wave modes and Love wave modes when the phase velocities of the Rayleigh waves and Love waves are different. But at periods where the Rayleigh and Love waves are degenerate, in the sense that their phase velocities are equal, the surface wave modes on a rotating Earth are a mix of Rayleigh wave and Love wave 


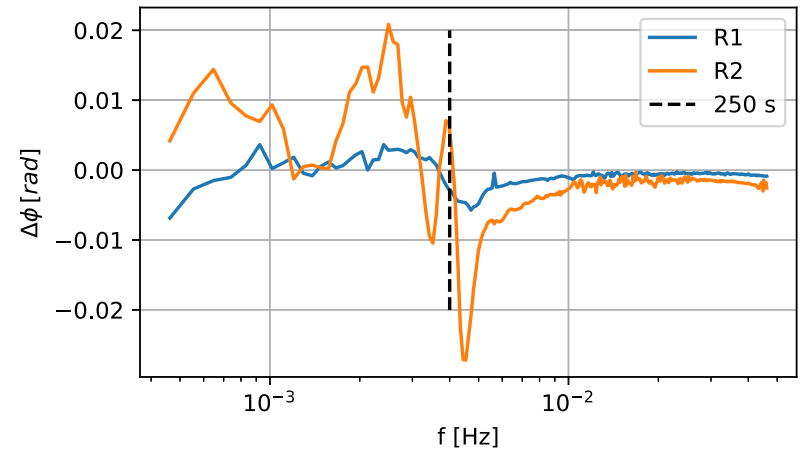

Figure 14. Phase differences between seismograms simulated with and without Earth's rotation averaged over all distances for the R1 and R2 segments of the Rayleigh wave.

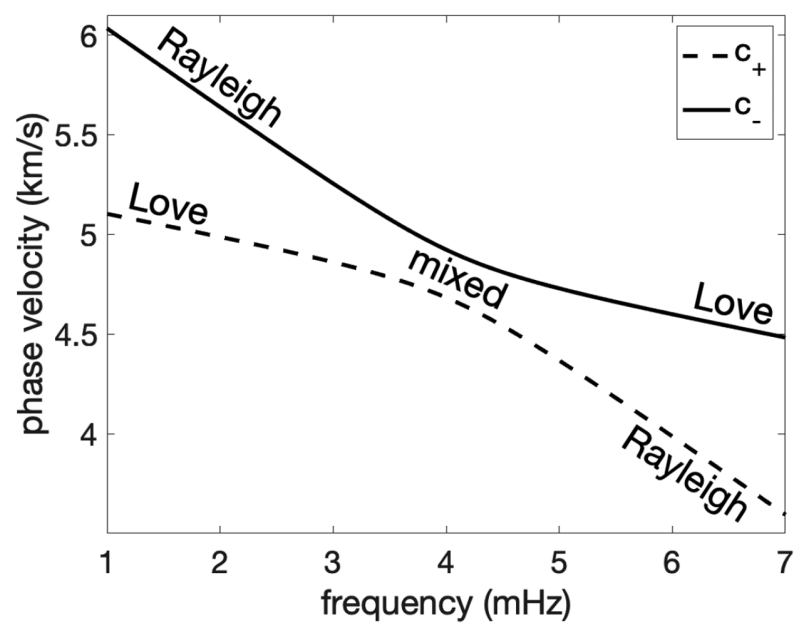

Figure 15. The phase velocity of the surface mode with the highest phase velocity (solid line) and the surface wave mode with the lowest phase velocity (dashed line) on a non-rotating Earth.

motion. This behavior is described by eqs (57)-(58) and fig. 3 of Snieder \& Sens-Schönfelder (2020). In the numerical example of that figure the Rayleigh waves and Love waves are degenerate for a period of $250 \mathrm{~s}$, or a frequency of $4 \mathrm{mHz}$. As shown in section 6 of Snieder \& Sens-Schönfelder (2020) the Rayleigh wave corresponds at frequencies much less than $4 \mathrm{mHz}$ to the mode with the highest phase velocity and for frequencies much larger than $4 \mathrm{mHz}$ to the mode with the lowest velocity, this behavior is sketched in Fig. 15. Close to the degenerate frequency (at $4 \mathrm{mHz}$ ) the eigenmodes on a rotating earth are a mix of Rayleigh wave and Love wave motion. As shown in Fig. 15 the phase velocity of Rayleigh waves 'jumps' from the phase velocity of the fastest mode to the phase velocity of the slowest mode. This gives rise to the growing jump in the phase difference for the Rayleigh wave that is shown in Fig. 14. The crossover of the dispersion curves sketched in Fig. 15 and the resulting jump in the phase shown in 14 occurs at a period of about $250 \mathrm{~s}$. This is the period for which Rayleigh waves and Love waves are observed to have the same phase velocity, see for example the phase velocities of Rayleigh and Love waves measured by Dziewonski \& Landisman (1970) that are indicated by dots in Fig. 2. The resonant propagating surface waves correspond to the coupling between the normal modes ${ }_{0} S_{32}$ and ${ }_{0} T_{31}$. A similar situation results from the coupling of modes with $l=12$ and $l=19$ at even longer periods (Masters et al. 1983).

\section{DISCUSSION}

The simulations presented here illustrate the effect of Earth's rotation on propagating surface waves and confirm the theoretical derivations of Snieder \& Sens-Schönfelder (2020) for the local coupling between radial and transverse displacement on a rotating Earth. One can discriminate two regimes of coupling: when the phase velocities are different and coupling is small on the one hand and the situation of degenerate phase velocities which causes strong coupling on the other hand. In globally averaged velocity models the dispersion curves of Love and Rayleigh waves cross at about $250 \mathrm{~s}$ period in the range of the degenerate normal modes ${ }_{0} T_{31}$ and ${ }_{0} S_{32}$ (Dahlen \& Tromp 1998). Phase velocities of fundamental Love and Rayleigh waves are also close in the frequency range of the microseism (10-20 s) and lateral heterogeneity may cause locally equal phase velocities. As Maupin (2004) points out, the velocity structures of oceanic or tectonically active regions can result in close phase velocities of different Love and Rayleigh modes allowing for strong local coupling.

If strong coupling occurs, it depends on location and azimuth of wave propagation (expressions 3 and 4). In general a component of the propagation vector along the direction of the rotation axis is required to couple radial and transverse motion. This is predominantly the case for propagation in the north-south direction in the equatorial region. There is no coupling along equatorial paths. For an initially pure Rayleigh wave, the conversion to Love wave occurs during propagation leading to a gradual buildup of transverse displacement. The rate of buildup is not constant but changes along a given path depending on the location, local amplitude (e.g. focusing at source and antipode) and local azimuth. The coupling of a Rayleigh wave to a Love wave by rotation perturbs the wavenumber of the Rayleigh wave. This leads to a repulsive perturbations of the hybrid multiplets of normal modes and repulsive phase perturbations of Rayleigh waves for frequencies above and below a frequency of degenerate phase velocity (Snieder \& Sens-Schönfelder 2020). In the wavefield simulations this can be observed by comparing waveforms with and without rotation and clearly shows the change in sign of the phase perturbation at the degenerate frequency.

Time-domain comparison of synthetic seismograms on a rotating Earth by Park \& Gilbert (1986) showed the azimuth dependence of the rotational coupling. Park (1986) compared synthetic seismograms in a laterally heterogeneous model with data and conclude that the effect of rotation is visible for favorable orientations of the source and nearly polar source-receiver paths in the form of well dispersed quasi-Love precursors to the Rayleigh wave arrivals similar to Fig. 9. The difficulty to observe the rotational coupling in time domain is partially due to the simultaneous excitation of Love and Rayleigh waves by earthquakes which complicates the isolation of rotational coupling during the propagation. Seismic interferometry (Curtis et al. 2006) makes it possible to create virtual sources, not only at locations defined by stations rather than real sources but moreover sources of different polarization can be created virtually. To demonstrate the local coupling we suggest to use the vectorial character of the coupling. For a roughly north-south oriented pair of stations with similar distances to the equator the waves propagating in opposite directions should attain coupled displacements of opposite sign. This means that the noise correlation $\left\langle Z_{n} E_{s}^{*}\right\rangle$ of the vertical component form the station on the Northern Hemisphere $\left(Z_{n}\right)$ with the east component of the station on the Southern Hemisphere $\left(E_{s}\right)$ should lead to the same waveform as $\left\langle Z_{s} E_{n}^{*}\right\rangle$, because the transverse directions for northward and southward propagation are oriented in the $E$ and $-E$ directions, respectively. Seismic interferometry thus 
makes is possible, in principle, to investigate rotational coupling by cross-correlating suitably chosen components of the wave motion.

\section{ACKNOWLEDGEMENTS}

Numerical simulations were performed on the Wendian highperformance computing system of Colorado School of Mines. We thank Ridvan Örsvuran for his help to run numerical simulations. The 3-D global seismic wave simulation package SPECFEM3D_GLOBE is freely available via Computational Infrastructure for Geodynamics (CIG; geodynamics . org). We thank Jeffrey Park and Gabi Laske for the critical and constructive comments of an earlier version of this manuscript. CS acknowledges support from the Alexander von Humboldt Foundation within a Feodor Lynen Research Fellowship.

\section{REFERENCES}

Aki, K. \& Richards, P.G., 2002. Quantitative Seismology, 2nd edn, University Science Books.

Ardhuin, F., Gualtieri, L. \& Stutzmann, E., 2015. How ocean waves rock the Earth: Two mechanisms explain microseisms with periods 3 to 300 s, Geophys. Res. Lett., 42(3), 765-772.

Ardhuin, F., Gualtieri, L. \& Stutzmann, E., 2019. Physics of ambient noise generation by ocean waves, in Seismic Ambient Noise, eds Nakata, N., Gualtieri, L. \& Fichtner, A., Cambridge Univ. Press.

Backus, B.Y.G. \& Gilbert, F., 1961. The rotational splitting of the free oscillations of the Earth, PNAS, 02(1), 362-371.

Backus, G.E., 1962. The propagation of short elastic surface waves on a slowly rotating Earth, Bull. seism. Soc. Am., 52(4), 823-846.

Bassin, C., Laske, G. \& Masters, G., 2000. The current limits of resolution for surface wave tomography in North America, EOS, Trans. geophys. Am. Soc., 81(48), F897.

Beghein, C., Resovsky, J. \& van Der Hilst, R.D., 2008. The signal of mantle anisotropy in the coupling of normal modes, Geophys. J. Int., 175(3), 1209-1234.

Ben-Menahem, A. \& Singh, S.J., 1981. Seismic Waves and Sources, Springer.

Curtis, A., Gerstoft, P., Sato, H., Snieder, R. \& Wapenaar, K., 2006. Seismic interferometry-turning noise into signal, Leading Edge, 25(9), 1082.

Dahlen, F. \& Smith, M., 1975. The influence of rotation on the free oscillations of the Earth, Philos. Trans. R. Soc. Lond., 279(1292), 583-629.

Dahlen, F.A., 1968. The normal modes of a rotating, elliptical Earth, Geophys. J. R. Astron. Soc., 16(4), 329-367.

Dahlen, F.A., 1969. The normal modes of a rotating, elliptical Earth - II nearresonance multiplet coupling, Geophys. J. R. astr. Soc., 18, 397-436.

Dahlen, F.A. \& Tromp, J., 1998. Theoretical Global Seismology, Princeton Univ. Press.

Deen, M., Wielandt, E., Stutzmann, E., Crawford, W., Barruol, G. \& Sigloch, K., 2017. First observation of the Earth's permanent free oscillations on ocean bottom seismometers, Geophys. Res. Lett., 44(21), 10 988-10996.

Dziewonski, A. \& Landisman, M., 1970. Great circle Rayleigh and love wave dispersion from 100 to 900 seconds, Geophys. J. R. astr. Soc., 19(1), 37-91.

Dziewonski, A.M. \& Anderson, D.L., 1981. Preliminary reference Earth model, Phys. Earth Planet. Inter., 25(4), 297-356.

Kennett, B.L., 1984. Guided wave propagation in laterally varying media I. Theoretical development, Geophys. J. R. astr. Soc., 79(1), 235-255.

Komatitsch, D. \& Tromp, J., 2002a. Spectral-element simulations of global seismic wave propagation - I. Validation, Geophys. J. Int., 149(2), 390412

Komatitsch, D. \& Tromp, J., 2002b. Spectral-element simulations of global seismic wave propagation - II. 3-D models, oceans, rotation, and selfgravitation, Geophys. J. Int., 150(1), 303-318.
Kurrle, D. \& Widmer-Schnidrig, R., 2008. The horizontal hum of the Earth: a global background of spheroidal and toroidal modes, Geophys. Res. Lett., 35(6), 1-5.

Kustowski, B., Ekström, G. \& Dziewoński, A.M., 2008. Anisotropic shearwave velocity structure of the earth's mantle: a global model, J. geophys. Res., 113(6), 1-23.

Landau, L.D., Lifshitz, 1959. Theory of Elasticity: Vol. 7 of Course of Theoretical Physics, Pergamon Press.

Laske, G. \& Masters, G., 1998. Surface-wave polarization data and global anisotropic structure, Geophys. J. Int., 132(3), 508-520.

Longuet-Higgins, M., 1950. A theory of the origin of microseisms, Philos. Trans. R. Soc. Lond., 243(857), 1-35.

Luh, P.C., 1974. Normal modes of a rotating, self gravitating inhomogeneous Earth, Geophys. J. R. astr Soc., 38(1), 187-224.

MacDonald, G. J.F. \& Ness, N.F., 1961. A study of the free oscillations of the Earth, J. geophys. Res., 66(6), 1865.

Masters, G., Park, J. \& Gilbert, F., 1983. Observations of coupled spheroidal and toroidal modes, J. geophys. Res., 88(B12), 10285-10298.

Maupin, V., 2004. Comment on 'The azimuthal dependence of surface wave polarization in a slightly anisotropic medium' by T. Tanimoto, Geophys. J. Int., 159(1), 365-368.

Nishida, K., 2014. Source spectra of seismic hum, Geophys. J. Int., 199(1), 416-429.

Nishida, K., Kobayashi, N. \& Fukao, Y., 2000. Resonant oscillations between the solid Earth and the atmosphere, Science, 287(5461), 2244-2246.

Nishida, K., Kawakatsu, H., Fukao, Y. \& Obara, K., 2008. Background Love and Rayleigh waves simultaneously generated at the Pacific Ocean floors, Geophys. Res. Lett., 35(16), 1-5.

Park, J., 1986. Synthetic seismograms from coupled free oscillations: effects of lateral structure and rotation, J. geophys. Res., 91(B6), 6441-6464.

Park, J., 1990. Observed envelopes of coupled seismic free oscillations, Geophys. Res. Lett., 17(10), 1489-1492.

Park, J. \& Gilbert, F., 1986. Coupled free oscillations of an aspherical, dissipative, rotating Earth: Galerkin theory, J. geophys. Res., 91(B7), $7241-7260$

Park, J. \& Yu, Y., 1993. Seismic determination of elastic anisotropy and mantle flow, Science, 261(5125), 1159-1162.

Rhie, J. \& Romanowicz, B., 2004. Excitation of Earth's continuous free oscillations by atmosphere-ocean-seafloor coupling, Nature, 431, 552556.

Rhie, J. \& Romanowicz, B., 2006. A study of the relation between ocean storms and the Earth's hum, Geochem. Geophys. Geosyst., 7(10), https://doi.org/10.1029/2006GC001274.

Sato, H., Fehler, M. \& Maeda, T., 2012. Seismic Wave Propagation and Scattering in the Heterogeneous Earth, 2nd edn, Springer.

Snieder, R., 1986.3-D linearized scattering of surface waves and a formalism for surface wave holography Roe1 Snieder, Geophys. J. R. astr. Soc, 84, 581-605.

Snieder, R. \& Sens-Schönfelder, C., 2020. Local coupling and conversion of surface waves due to Earth's rotation. Part 1: theory, Geophys. J. Int, $10.1093 /$ gji $/$ ggaa587.

Snieder, R., Sens-Schönfelder, C. \& Ruigrok, E., 2016a. Elastic-wave propagation and the Coriolis force, Phys. Today, 69(12), 90-91.

Snieder, R., Sens-Schönfelder, C., Ruigrok, E. \& Shiomi, K., 2016b. Seismic shear waves as foucault pendulum, Geophys. Res. Lett., 43, 2576-2581.

Tanimoto, T., 2001. Continuous free oscillations: atmosphere-solid Earth coupling, Annu. Rev. Earth planet. Sci., 29(1), 563-584.

Tanimoto, T., Hadziioannou, C., Igel, H., Wassermann, J., Schreiber, U. \& Gebauer, A., 2015. Seasonal variations in the Rayleigh-to-Love wave ratio in the secondary microseism from colocated ring laser and seismograph, Geophys. Res. Lett., 42, 2650-2655.

Webb, S.C., 2007. The Earth's 'hum' is driven by ocean waves over the continental shelves, Nature, 445(7129), 754-756.

Zürn, W., Laske, G., Widmer-Schnidrig, R. \& Gilbert, F., 2000. Observation of coriolis coupled modes below $1 \mathrm{mHz}$, Geophys. J. Int., 143(1), 113118. 